\title{
Metodologías analíticas utilizadas actualmente para la determinación de mercurio en músculo de pescado
}

\author{
Analytical methodologies currently used for the determination of mercury in fish muscle
}

\author{
Ingrid Ruiz Chaves ${ }^{1}$ \\ Recibido: 18/8/2015 / Aprobado: 12/4/2016
}

Resumen

El mercurio es un metal pesado con tiene efectos tóxicos, una de las vías de ingreso en los humanos es por el consumo de pescado, debido al riesgo representado para la salud, existe un gran interés en el desarrollo de técnicas analíticas sensibles y fiables para su determinación. Para la determinación del mercurio total existe una gran variedad de metodologías que proporcionan información parcial acerca de su impacto en la salud humana y el medio ambiente, como consecuencia se han desarrollado técnicas capaces de separar e identificar las diversas especies de mercurio para poder evaluar los distintos grados de impacto.

El

presente trabajo realiza una propuesta del principio básico, ventajas e inconvenientes de las principales técnicas en la determinación de mercurio total y metilmercurio en matrices alimentarias (músculo de pescado) empleadas por métodos analíticos directos e indirectos, tales como: la espectrometría de absorción atómica de vapor frío (CV AAS), vapor frío espectrometría de fluorescencia atómica (CV AFS), plasma acoplado inductivamente-espectrometría de masas (ICP-MS) y analizador de mercurio. También se presenta un breve texto con el uso de técnicas cromatográficas para el análisis de especiación del mercurio orgánico.

Se han desarrollado muchas técnicas analíticas para determinar el mercurio, las cuales han logrado tener un mayor grado de especificidad y selectividad con equipos muy sensibles, para determinar concentraciones en el de nivel ultratrazas y lograr la identificación y separación de los diferentes compuestos del mercurio, para garantizar el riesgo asociado al consumo de pescado.

Palabras claves: mercurio, (metil) mercurio, músculo de pescado, técnicas analíticas, espectroscopia, cromatografía.

\section{Abstract}

Mercury is a heavy metal that has toxic effects. Fish consumption is one of the main sources of Human exposure to mercury. There is great interest in the development of sensitive and reliable analytical techniques to determine mercury levels in humans due to health risks. There are a variety of methodologies that provide partial information about the impact of mercury levels on human health and the environment. Consequently, techniques to separate and identify the various species of mercury have been developed to assess the different degrees of the impact. This article presents a proposal of the basic principle, advantages, and disadvantages of the main techniques for the determination of total mercury and methylmercury in food matrices (fish muscle) employing direct and indirect analytical methods such as: Cold Vapor-Atomic Absorption Spectrophotometry (CVAAS), Cold Vapor Atomic Fluorescence Spectroscopy (CVAFS), Inductively Coupled Plasma Mass Spectrometry (ICP-MS), and Mercury Analyzer. A short text on the use of chromatographic techniques for speciation analysis of organic mercury is included. There has been a great development in analytical techniques to determine mercury levels. These techniques have achieved a greater degree of specificity and selectivity with very sensitive equipment for the determination of ultra-trace level concentrations, and the identification and separation of the different compounds of mercury in order to confirm the risk associated with fish consumption.

Keywords: mercury, methylmercury, fish muscle, analytical techniques, spectroscopy, chromatography.

\footnotetext{
${ }^{1}$ Bachiller en Laboratorista Químico. Analista de Laboratorio SENASA-LANASEVE. Ministero de Agricultura y Ganadería. Universidad de Costa Rica. Sede de Occidente, Recinto de Grecia. Correo elctrónico imruizch@hotmail.com
} 


\section{Introducción}

El mercurio ( $\mathrm{Hg}$ ) es un metal que en concentraciones de traza es altamente tóxico y dañino para los ecosistemas y para los seres humanos, de acuerdo con Fernández et al. (2005), citado en Pérez et al. (2012). Este metal es fácilmente metilado por microorganismos, se bioacumula en la biota y se biomagnifica en la cadena alimentaria. (Bustamante et al., 2006, citado en Pérez et al., 2012).

Las principales formas químicas de mercurio son: mercurio elemental ( $\mathrm{Hg} 0)$, mercurio inorgánico divalente $\left(\mathrm{Hg}^{+2}\right)$, metil mercurio $\left(\mathrm{CH}_{3}\right.$ $\left.\mathrm{Hg}^{+}\right)$y dimetilmercurio $\left(\left(\mathrm{CH}_{3}\right)_{2} \mathrm{Hg}\right)$. (Cornelis et al. 2005, citado en Ferreira et al., 2014).

Cabe destacar que los efectos tóxicos del Hg dependerán de la forma química en la que se encuentre, los del metilmercurio ( $\mathrm{MeHg}$ ) son más tóxicos que el mercurio elemental y sus sales inorgánicas, pues es absorbido eficientemente en el tracto gastrointestinal, pasa de la sangre al cerebro y traspasa la barrera de la placenta, en el caso de los fetos, por lo cual afecta el sistema nervioso central con daños irreversibles. (Pérez et al, 2012).

En la actualidad, se considera que el consumo de pescado constituye la principal fuente de exposición humana al mercurio (EFSA, 2004). Según Sánchez (2010), el control del Hg se ha incrementó significativamente desde el primer accidente bien documentado, ocurrido por intoxicación con este metal en la Bahía de Minamata, Japón, luego del cual, la Organización Mundial de la Salud, agencias de protección ambiental y entes gubernamentales de diferentes países establecieron alternativas para el control y monitoreo de este elemento en el ambiente. Por lo tanto, no solo el seguimiento de las concentraciones totales de mercurio en el medio ambiente es suficiente; el análisis de especiación proporciona información más útil para evaluar los riesgos de toxicidad del mercurio en la salud y además entender los ciclos biogeoquímicos de los compuestos de mercurio (Gao et al., 2012).

La presencia de mercurio en los peces se presenta debido a la capacidad de estos de acumular el metal, por su gran solubilidad en los lípidos. Se ha determinado que la concentración de Hg en peces puede ser 10000 mil a 100000 mil veces mayor a la encontrada en el propio sustrato o alimento. La ingesta de pescado con altas concentraciones de $\mathrm{Hg}$ produce graves daños en el organismo humano (Wittwer, 2012).

Una vez absorbido el mercurio por el organismo, es distribuido uniformemente, pero la mayor parte en el cerebro, hígado y riñón. Los principales efectos tóxicos se producen sobre el sistema nervioso, especialmente en el sistema nervioso central. Hay que destacar la especial sensibilidad presentadas en los niños y las mujeres embarazadas, afectados por los mismos efectos cuando sufren una dosis cinco veces menor (Camaren, 2003).

El Codex Stan 1993-1995, establecido por la Organización para la Agricultura y la Alimentación de las Naciones Unidas (FAO), de la Organización Mundial de la Salud OMS (OMS), establece los niveles máximos de metilmercurio en el pescado y los peces depredadores $\left(0,5\right.$ y $1 \mathrm{mg} \mathrm{kg}^{-1}$, respectivamente ) (CODEX, 2009).

Existe un gran interés en el desarrollo de técnicas analíticas sensibles y fiables para determinar el contenido de mercurio, debido a los riesgos para la salud. Diversas técnicas de detección están actualmente disponibles, como la espectrometría de absorción atómica de vapor frío (CV AAS), plasma acoplado inductivamente espectrometría de emisión óptica (ICP-OES), espectrometría de absorción atómica electrotérmica (ET AAS), Espectrometría de fluorescencia atómica (CV-AFS), Espectrometría de masas (ID-ICP / MS).

A pesar de los avances instrumentales, algunas técnicas no tienen la sensibilidad y selectividad adecuada para la determinación de este elemento en un gran número de matrices. Por lo tanto, se requieren generalmente antes del análisis la separación y el enriquecimiento previo del analito. Diferentes materiales y estrategias de análisis se han desarrollado para este fin (Lemos et al., 2014).

El analito, por ser un metal pesado, tiene la característica de una gran estabilidad, lo cual permite que la matrices sean fortificadas previamente antes 
de realizar la metodología, aunque queda sujeto al grado de cuantificación y equipo el utilizar lo que determinará cuáles materiales utilizar y la forma de la extracción. Todas las tecnologías utilizadas tienen buena sensibilidad y permiten cuantificar el mercurio en niveles traza y ultratraza. Sin embargo, la elección de estas metodologías y tecnologías involucra aspectos que abarcan desde el costo inicial de las herramientas espectroanalíticas, la cantidad de reactivos usados en las etapas de apertura hasta los niveles de interferencias espectrales (de Jesús et al., 2011).

Según Rodríguez (2009), en la mayoría de los métodos, el mercurio en la muestra se reduce al estado elemental. Algunos métodos requieren la predigestión de la muestra antes de la reducción, esto se lleva a cabo con digestiones en microondas, donde la muestra se trata antes para eliminar posibles interferencias. En todas las fases de la preparación y del análisis de la muestra, la posibilidad de contaminación del mercurio encontrado naturalmente en el ambiente debe ser considerada, por tanto, tienen que seguirse estándares rigurosos para prevenirla siempre que sea posible; el instrumental y el equipo en contacto con la muestra no podrán contener los metales objeto de la determinación y deberán estar hechos de materiales inertes, los instrumentos cortantes deben ser de acero inoxidable de alta calidad y todo el material por utilizar debe limpiarse previamente con ácido, para minimizar el riesgo de contaminación, esto según la Comisión Europea en su Decisión 333/2007/CE.

El objetivo de esta revisión es explicar las técnicas con más frecuencia, en la Tabla 1 se presentan los métodos disponibles para la determinación de mercurio con los límites de detección reportados, esto como base para la selección, de acuerdo con los niveles máximos permitidos por la legislación. Cabe destacar que los métodos más utilizados emplean equipos instrumentales, esto por el nivel de sensibilidad requerido para determinar y el tipo de matriz.

Tabla 1: Métodos más frecuentemente utilizados para la cuantificación de mercurio y sus respectivos límites de detección

\begin{tabular}{ll}
\hline Método & $\begin{array}{l}\text { Límite de detección } \\
\text { reportado Mercurio total }\end{array}$ \\
\hline Método colorimétrico de ditizona & $0,01-0,1 \mathrm{mg} / \mathrm{g}$ \\
\hline Espectrometría de absorción atómica - horno de grafito (GF AAS) & $1 \mathrm{ng} / \mathrm{g}, 0,01-1 \mathrm{ng} / \mathrm{g}, 0,001-0,01 \mathrm{ng} / \mathrm{g}$ \\
\hline Análisis por activación neutrónica- instrumental (INAA) & $1-10 \mathrm{ng} / \mathrm{g}$ \\
\hline Radioquímica (RNAA) & $0,01-1 \mathrm{ng} / \mathrm{g}$ \\
\hline Cromatografía gaseosa - detector de captura electrónica & $0,01-0,05 \mathrm{ng} / \mathrm{g}$ \\
\hline Detector de emisión atómica & $\sim 0,05 \mathrm{ng} / \mathrm{g}$ \\
\hline Espectrometría de masas & $0,1 \mathrm{ng} / \mathrm{g}$ \\
\hline CVAAS / CVAFS & $0,01-0,05 \mathrm{ng} / \mathrm{g}$ \\
\hline Cromatografía líquida de alta eficiencia - detector de UV & $1 \mathrm{ng} / \mathrm{mL}$ \\
\hline CVAAS & $0,5 \mathrm{ng} / \mathrm{mL}$ \\
\hline CVAFS & $0,08 \mathrm{ng} / \mathrm{mL}$ \\
\hline Electroquímico & $0,1-1 \mathrm{ng} / \mathrm{mL}$ \\
\hline Plasma acoplado inductivamente-espectrometría masas (ICP MS) & $0,01 \mathrm{ng} / \mathrm{mL}$ \\
\hline Espectrometría de emisión atómica (ICP AES) & $2 \mathrm{ng} / \mathrm{mL}$ \\
\hline Espectrometría foto-acústica & $0,05 \mathrm{ng}$ \\
\hline Fluorescencia de rayos X & $5 \mathrm{ng} / \mathrm{g}-1 \mathrm{mg} / \mathrm{g}$ \\
\hline Métodos electroquímicos & $0,1-1 \mathrm{mg} / \mathrm{g}$ \\
\hline
\end{tabular}


Un factor importante para garantizar la calidad y confiabilidad de los resultados es elegir el tipo de matriz biológica o ambiental por evaluar y cómo los niveles de mercurio en esta pueden estar asociados con las especies químicas que se van a cuantificar a través de métodos directos o indirectos.

Algunos tipos de muestras biológicas y ambientales presentan mercurio en niveles traza que exigen usar equipos con detectores altamente sensibles, procesos delicados de preconcentración y reactivos apropiados con alto grado de pureza (Mailman et al.,2006),(Jia et al.,2011).

Las metodologías para determinar este metal deben ser muy selectivas, específicas y sensibles, cuyo rango de resultados pueda involucrar resultados en niveles muy bajos, por lo tanto se debe garantizar que estas se encuentren validadas y acreditadas bajo la norma INTE IEC/17025:2015, con el fin de garantizar resultados confiables, los cuales se puedan usar en la vigilancia ambiental del mercurio y garantizar que el alimento (pescado) se puede consumir sin ningún riesgo a la salud.

\section{Métodos analíticos para determinar mercurio}

\section{A. Determinación de mercurio al emplear análisis indirecto}

La determinación de las concentraciones de especies de mercurio en muestras biológicas (pescado) es un reto debido a las bajas concentraciones de este elemento, y la evaluación de la toxicidad es difícil porque se requiere cuantificar cada una por separado. Varios estudios en la literatura describen métodos para la determinación de las concentraciones de especies de mercurio al utilizar técnicas de espectrometría atómicas (Moraes et al., 2013).

\section{Espectroscopia de absorción atómica con vapor frío (CVAAS)}

El método más utilizado para la determinación del mercurio en cualquier medio ha sido la espectroscopia de absorción atómica (AAS) con la técnica del vapor frío. (Panichev y Panicheva, 2015). Esta técnica permite la determinación directa con la única condición de que el mercurio contenido en las muestras líquidas, normalmente en forma iónica como $\mathrm{Hg} 2+$, sea reducido al estado metálico $\mathrm{Hg}^{0}$. Posteriormente, el vapor formado se arrastra por un gas inerte hacia una celda de cuarzo en la que se produce el proceso de la absorción atómica. Las muestras sólidas se digieren antes para transformar todas las especies de $\mathrm{Hg}$ (inorgánicas y orgánicas) a $\mathrm{Hg} 2+$, ya que esta es la única especie capaz de generar el vapor de mercurio atómico (González et al., 2014).

Dentro de las desventajas se puede citar que el método consume tiempo y es complicado por la posibilidad de pérdidas por volatilización o digestión incompleta, así como la contaminación de las muestras (Kuboyama et al., 2005).

Se denota que de todas las técnicas analíticas utilizadas esta es la más empleada, por realizar etapas muy sencillas de extracción, además de cuantificar satisfactoriamente el mercurio en muestras de pescado.

\section{Espectrometría de masas (ICP-MS)}

La introducción del ICP-MS en los laboratorios permite realizar un análisis simultáneo de casi todos los elementos del sistema periódico, obtener bajos límites de detección y la posibilidad de estudiar diferentes isotopos para un mismo elemento. Sin embargo, esta técnica tiene algunas limitaciones importantes para el estudio de muestras, debido a las altas concentraciones de constituyentes orgánicos e inorgánicos que producen efectos de matriz e interferencias espectrales causadas por la formación de iones poliatómicos. La introducción de las celdas de reacción dinámica/colisión como sistema para el control de las interferencias espectrales ha ayudado a resolver este problema, y esto hace posible que 
actualmente el ICP-MS pueda ser usado en los laboratorios en análisis de rutina (González et al., 2014).

Hoy, el uso de ICP-MS se está volviendo más común en el análisis de laboratorio de los alimentos (Leblanc et al., 2005). En comparación con GF AAS o ICP-OES, esta técnica tiene algunas ventajas, como la capacidad de medición simultánea con varios elementos junto con los límites de detección muy bajos (Ammann, 2007 y Parsons y Barbosa, 2007). Además, ofrece un rango dinámico lineal amplio que permite la determinación de elementos mayores y traza en la misma inyección de la muestra (Ammann, 2007 y Parsons y Barbosa, 2007). Además, en comparación con ICP-OES, ICP-MS ofrece una interpretación espectral más simple y la información isotópica.

Sin embargo, ICP-MS tiene algunas limitaciones. Para el análisis de muestra de alimento, la alta concentración de matriz orgánica a menudo resulta en interferencias de la matriz o interferencias espectrales de iones poliatómicos. Estos efectos pueden ser eliminados o minimizados mediante el uso de isótopos alternativos o ecuaciones de corrección de interferencia (Nardi et al., 2009).

\section{Espectrometría de masas con plasma acoplado por inducción (ID-ICP/ MS)}

Estas técnicas analíticas son adecuadas para análisis de rutina debido a su alta sensibilidad y precisión razonable. En general, es bien conocido que la dilución de plasma inductivamente acoplado isótopo / espectrometría de masas (ID-ICP / MS) tiene un alto potencial para análisis de rutina de elementos traza si la exactitud de los resultados es de importancia analítica predominante. Aunque el método ID-ICP / MS ha sido ampliamente utilizado para la determinación de elementos traza en diversas matrices, solo unas pocas aplicaciones han sido reportadas para la determinación de $\mathrm{Hg}$. El problema en el análisis de $\mathrm{Hg}$ es que el efecto de memoria aumenta los recuentos en blanco y empeora el funcionamiento analítico de ICP-MS (Youshinaga y Morita, 1997).
Otro problema es la posibilidad de pérdida de Hg durante el procedimiento de descomposición de la muestra debido a su volatilidad (Lee et al., 2005).

\section{Espectrometría de absorción atómica con horno de grafito (GFAAS)}

Un aspecto importante del análisis de muestreo sólido directo de mercurio con atomizador electrotérmico combinado con AAS es la posibilidad de operar con programas rápidos. Con la aplicación de estos programas los tiempos de secado y pirólisis se minimizan o la etapa de pirólisis se elimina y el tiempo de análisis general se acorta de manera significativa, con un aumento del rendimiento analítico (Tuzen et al., 2009). En el caso particular de análisis de mercurio, el programa corto tiene una ventaja adicional: la eliminación de la etapa de pretratamiento térmico disminuye la posibilidad de pérdidas de mercurio. El reto importante en la determinación de las concentraciones de mercurio con el uso de GFAAS está impidiendo la pérdida de mercurio por volatilización durante el secado y la pirólisis de la muestra. Esta pérdida de analito puede prevenirse con el uso de modificadores químicos estabilizadores del mercurio antes de la atomización. Varios tipos de modificadores químicos para la determinación de las concentraciones de mercurio han sido reportados en la literatura (Moraes et al., 2013).

Esta metodología tiene un nivel muy alto de sensibilidad, se utiliza para monitorizar los niveles de mercurio en muestras de tejido de músculo de pescado pues se pueden determinar concentraciones a nivel de $\mathrm{ppb}$.

\section{Espectrometría de fluorescencia atómica (CV-AFS)}

La cuantificación de mercurio total en los productos alimenticios puede ser realizada por vapor frío espectrometría de fluorescencia atómica (CVAFS). Su sensibilidad inherente ofrece niveles de detección muy bajos y amplio rango dinámico lineal. Esto hace a CV-AFS una poderosa herramienta analítica claramente ventajosa sobre técnicas de 
absorción atómica. Además, la instrumentación requerida es mucho más simple que la necesaria para las técnicas de espectrometría de masas (Martínez et al., 2015).

Aunque esta técnica se utiliza en matrices de alimentos, en la revisión se encontró la evaluación de diferentes métodos por CV-AFS para determinar $\mathrm{Hg}$ en algas marinas (Martinez et al., 2015).

\section{B. Determinación de mercurio en matrices alimentarias con análisis directo}

Analizador de Mercurio (DMA)

El uso del analizador de mercurio directo (DMA) es una de las buenas alternativas propuestas para la determinación de trazas de mercurio en matrices complejas, como los alimentos (Butala et al., 2006, Ipolyi et al., 2004,Ikem et al., 2005, Carbonell et al., 2009 ,Torres et al., 2012 y Viera et al., 2014). Este equipo se puede emplear para el análisis de muestras sólidas y líquidas y que no requieren ningún tratamiento previo. Se opera sobre una base de muestra de la descomposición térmica, seguido por reducción catalítica de mercurio y en un sistema de amalgama oro para la captura de vapor de mercurio para, posteriormente, la desorción de mercurio y como técnica analítica espectrometría de absorción atómica. La eficiencia de un análisis químico al usar DMA depende mucho de la homogeneidad de la muestra que se está procesando. En general, las curvas de calibración se establecen utilizando materiales de referencia certificados pero, con el tiempo, se pueden realizar también por la técnica de calibración externa al emplear soluciones acuosas estándares (Carbonell, 2009 y Torres et al., 2012). De esta manera, el DMA se ha utilizado para la determinación de trazas de mercurio en varias matrices (Hight et al., 2006, Butala et al., 2006). El único inconveniente del método DMA es la cantidad de muestra que se limita a $100 \mathrm{mg}$, sin embargo el método DMA ofrece límites de detección y cuantificación más bajos que el método CV AAS (Vieira et al., 2014).

\section{c. Cuantificación del mercurio orgánico (especiación)}

Las fuentes de exposición y la toxicidad de las especies de mercurio son muy diferentes. Por tanto, es de gran importancia el poder disponer de métodos analíticos capaces de diferenciar las distintas especies del mercurio (González et al., 2014).

En las últimas décadas, se desarrollaron muchas metodologías de especiación del mercurio que usaban tecnologías como cromatografía líquida con espectrometría de masas (LC-MS), cromatografía gaseosa con espectrometría de masas (CG-MS) y cromatografía gaseosa con detector de captura de electrones (GCECD) (de Jesús et al., 2011).

También se lograron avances significativos en el desarrollo de sistemas híbridos y sistemas on-line en flujo, con énfasis en tecnologías que combinan separaciones cromatográficas y detección por métodos espectroanalíticos, como, por ejemplo, LC-CV-AAS, LC-ICP-MS, LC-CV-FAS, CG-FAS (de Jesus et al., 2011).

Se están perfeccionando los sistemas que usan cromatografía líquida o gaseosa acoplados a espectrómetros de masa. Estos han demostrado alta versatilidad para identificar y cuantificar especies organomercuriales (metilmercurio, etilmercurio dimetilmercurio,fenilmercurio, etc) (de Jesus et al., 2011).

Según Quevauviller et al., 2000, una ventaja obvia de los métodos cromatográficos es la capacidad de distinguir entre diferentes especies de mercurio en las muestras. Sin embargo, una desventaja común en estos métodos es que implican procedimientos de pretratamiento complejos y tediosos para la separación de mercurio orgánico ( $\mathrm{MeHg}$ en muestras biológicas) de la matriz de la muestra por diferentes métodos, tales como destilación, extracción - digestión disolvente alcalino y la digestión ácida -extracción solvente.

En un estudio realizado por Zhang y Adeloju (2012), con esta metodología se logró extraer y separar el metil mercurio del mercurio total, para así establecer las proporciones de cada especie y su aporte. 


\section{Conclusiones}

Es de gran importancia el desarrollo de métodos para la determinación de trazas de mercurio en muestras de pescado, como uno de los alimentos con mayor riesgo de exposición, por su alta toxicidad. Se han logrado practicar metodologías altamente sensibles y específicas en el análisis de este metal y sus diferentes especies.

La espectrometría de absorción atómica de vapor frío (CV AAS) ha sido el método más utilizado, debido a su velocidad y simplicidad, relativa libertad de interferencias, y alta sensibilidad. Otras de las técnicas citadas tienen un grado muy alto de sensibilidad, pero presentan algunas limitaciones como alta volatilidad del mercurio y sus compuestos, etapas previa de mineralización y el uso de modificadores químicos entre otros. La determinación del mercurio total ya no es una metodología completamente aceptable, ya que proporciona información parcial acerca de los niveles de metil mercurio en pescado y no se logra evaluar el impacto en la salud humana y el medio. Como consecuencia, se han desarrollado técnicas que son capaces de separar e identificar las diversas especies de mercurio. Se han desarrollado equipos y metodologías tan selectivas y específicas para garantizar que el pescado consumido no tiene ningún tipo de riesgo a la salud, pues logran con facilidad separar las especies orgánicas y inorgánicas del mercurio, y así, con los resultados obtenidos, verificar el cumplimiento con la legislación vigente.

Los métodos más utilizados en la determinación del mercurio son los métodos cromatográficos y los métodos espectrométricos; la ventaja de los cromatográficos es su especificidad, pues tienen la capacidad de distinguir entre diferentes especies de mercurio en las muestra. Se han desarrollado métodos directos que ha sido una buena opción para la determinación en muestras de alimentos sólidos y líquidos.

\section{Referencias bibliográficas}

Ammann A. (2007). ICP-MS (ICP MS): una herramienta versátil. Diario de Espectrometría de Masas, (42), 419-427

Bustamante, P.; et al. (2005). Total and organic $\mathrm{Hg}$ concentrations in cephalopods from the North Eastern Atlantic waters: Influence of geographical origin and feeding ecology. Science of The Total Environment. (368), 58596.

Butala S.J.M, Scanlan L.P., Chaudhuri S.N. (2006). A detailed study of thermal decomposition, amalgamation/atomic absorption spectrophotometry methodology for the quantitative analysis of mercury in fish and hair J. Food Prot., 69, 2720-2728

Camaren, (2003). Foro de los Recursos Hídricos. Segundo encuentro nacional, Quito, Ecuador.

Campbell M, Vermeir, G . (1992) Dams Influence of chemical species on the determination of mercury in a biological matrix (cold muscle) using inductively coupled plasma mass spectrometry. J. Anal. At. Spectrom., 7, p. 617

Carbonell G., Bravo J.C., Fernandez C., Tarazona J.V. (2009). A new method for total mercury and methyl mercury analysis in muscle of seawater fish Bull. Environ. Contam. Toxicol, $83,210-213$

Cornelis R., Caruso JA, Crews H., Heumann KG. (2005). Manual de especiación elemental,Handbook of Elemental especiación II: Especies en el Medio Ambiente, Alimentación, Medicina y salud en el trabajo.John Wiley \& Sons, Chichester, Inglaterra 
CODEX STAN 193-1995(2009). Norma General del Codex para los contaminantes y las toxinas presentes en los alimentos y piensos CE, el Reglamento modifica el Reglamento (CE) no 1881/2006 por el que se establecen tolerancias máximas de determinados contaminantes en los productos alimenticios. Reglamento $\mathrm{N}^{\circ}$ 629/2008 de la Comisión, DO N ${ }^{\circ} 364$, de 20.12.2006, 2008

de Jesus, I. M., Paniagua, J. C., Uribe, M. V., \& de Oliveira, M. L. (2011) Capitulo 6: Metodologías de análisis de mercurio. COOPERACIÓN TÉCNICA ENTRE BRASIL, BOLIVIA Y COLOMBIA: Teoría y Práctica para el Fortalecimiento de la Vigilancia de la Salud de Poblaciones Expuestas a Mercurio, 47.

European Food Safety Authority (EFSA). (2004). Opinion of the Scientific Panel on Contaminants in the Food

Fernández-Martínez, R., Rucandio, M. I. (2005). Study of the suitability of $\mathrm{HNO}$ and $\mathrm{HCl}$ as extracting agents of mercury species in soils from cinnabar mines. Analytical and Bioanalytical Chemistry (381), 1499-506.

Ferreira, S., Lemos, V., Laiana, S., Queiroz, A., Souza, A., Silva, E., dos Santos, W., das Virgens, C. (2015). Analytical strategies of sample preparation for the determination of mercury in food matrices. A review Microchemical Journal (121)227-236

Gao, Y., Shi, Z., Long, Z., Wu, P., Zheng, Ch., Hou, $X$. (2012). Determination and speciation of mercury in environmental and biological samples by analytical atomic spectrometry. Microchemical Journal (103) 1-14
González-E, M., Bodas-Pinedo, A., Martínez-García, M. J., Trasobares-Iglesias, E. M., BermejoBarrera, P., Ordóñez-Iriarte, J. M., \& Pascual, A.C. (2014). Metilmercurio: recomendaciones existentes. Métodos de análisis e interpretación de resultados. Evaluación económica. Nutrición Hospitalaria,31(n01).

Hight, S., Cheng, J. (2006). Determination of methylmercury and estimation of total mercury in seafood using high performance liquid chromatography (HPLC) and inductively coupled plasma-mass spectrometry (ICPMS): Method development and validation. Analytica Chimica Acta (567) 60-170

Ipolyi, I., Massanisso, P., Sposato, S., Fodor, P., Morabito, R. (2004). Concentration levels of total and methylmercury in mussel samples collected along the coasts of Sardinia Island (Italy). Anal. Chim. Acta, (505) 145-151

Ikem A., Egiebor, N.O. (2005) Assessment of trace elements in canned fishes (mackerel, tuna, salmon, sardines and herrings) marketed in Georgia and Alabama (United States of America) J. Food Compos. Anal., (18), 771-787

Jia, X., Han, Y., Liu, X., Duan, T., Chen, H. (2011). Speciation of mercury in water samples by dispersive liquid-liquid microextraction combined with high performance liquid chromatographyinductively coupled plasma mass spectrometry. Spectrochimica Acta Part $B$; (66), 88-92

Kuboyama, K., Sasaki, N., Nakagome, Y., Kataoka, M. (2005), Digestión húmeda.Analytical Chemistry, 184-191

Leblanc, Guerin T., Noel L.,. Calamassi-Tran G, Volatier JL, Verger P. (2005) Estimaciones de la exposición dietética de 18 elementos de la primera estudio dieta total Francés. Aditivos y Contaminantes de los Alimentos, 22, pp. 624-641 
Lee, S., Suh, J. (2005). Determination of mercury in tuna fish tissue using isotope dilutioninductively coupled plasma mass spectrometry, Microchemical Journal (80),233-236

Lemos, V., dos Santos, L. (2014). A new method for preconcentration and determination of mercury in fish, shellfish and saliva by cold vapour atomic absorption spectrometry Food Chemistry (149), 203-207

Mailman, M., Stepnuk, L., Cicek, N., Bodaly, RA. (2006). Strategies to lower methyl mercury concentrations in hydroelectric reservoirs and lakes: A review. Science of the Total Environment, (368), 224- 235.

Martinez, R., Rucandio, I., Pinilla, I., Borlag, F., Garcia, F., Larrea, M. (2015). Evaluation of different digestion systems for determination of trace mercury in seaweeds by cold vapour atomic fluorescence spectrometry Journal of Food Composition and Analysis (38), 7-12

Moraes, P., Santos, F. , Cavecci, B., Padilha, C., Viera, J., Roldan, P., Padilha, P. (2013). GFAAS determination of mercury in muscle samples of fish from Amazon, Brazil Food Chemistry (141), 2614-2617

Nardi, E., Evangelista, F., Tormen L., Saint 'Pierre, T., Curtius, A., de Souza, S., Barbosa, Jr. F. (2009). The use of inductively coupled plasma mass spectrometry (ICP-MS) for the determination of toxic and essential elements in different types of food samples Food Chemistry (112) 727-732

Panichev, N. y Panicheva, S. (2015). Determination of total mercury in fish and sea products by direct thermal decomposition atomic absorption spectrometry Food Chemistry 166 432-441
Parsons PJ , Barbosa, F. (2007). Espectrometría y las tendencias en la medicina clínica de laboratorio Atómica Spectrochimica. Acta, parte B, 62, 992-1.003

Pérez D. E., \& Peñuela, G. A. (2012). Trascendencia del metilmercurio en el ambiente, la alimentación y la salud humana.

Quevauviller P, Filippelli M, Horvat M, (2000). Method performance evaluation for methylmercury determination in fish and sediment. Trends Anal. Chem., (19), 157

REGLAMENTO (CE) No 333/2007 DE LA COMISIÓN de 28 de marzo de 2007 por el que se establecen los métodos de muestreo y análisis para el control oficial de los niveles de plomo, cadmio, mercurio, estaño inorgánico, 3-MCPD y benzopireno en los productos alimenticios.

Rodríguez, LH. (2009).Comparación de dos métodos de determinación de mercurio total en cabello por espectroscopías de absorción atómica con generador de hidruros y diferencial de efecto zeeman con pirolizador. Tesis Maestría en Toxicología, Universidad Nacional de Colombia.

Sánchez. Juan C. (2010) Metodologías analíticas para la determinación de metales tóxicos en muestras de interés ambiental. Tesis Magister en Química, Universidad Nacional de Colombia.

Torres D.P., Martins-Teixeira, M.B. (2012). Method development for the control determination of mercury in seafood by solid-sampling thermal decomposition amalgamation atomic absorption spectrometry (TDA AAS) Food Addit. Contam., Part B,( 29), 625-632 
Tuzen, M., KaramanI, Citak D., Soylak, M. (2009), Determination of total mercury for marine environmental monitoring studies by solid sampling continuum source high resolution atomic absorption spectrometry. Food and Chemical Toxicology (47)1648-1652

Vieira H.P., Nascentes C.C., Windmoller C.C. (2014).Development and comparison of two analytical methods to quantify the mercury content in honey J. Food Compos. Anal., (34), $1-6$

Wittwer Paulina, P.(2012). Efecto de la cocción sobre la concentración de mercurio $(\mathrm{Hg})$ y selenio (Se) en productos pesquero. Tesis Licenciatura en Ciencias de los Alimentos, Universidad Astral de Chile.

Youshinaga J, Morita M. (1997). Determination of mercury in biological and environmental samples by inductively coupled plasma mass spectrometry with the isotope dilution technique. J. Anal. At. Spectrom., 12 p. 417

Zhang Y., Adeloju S. (2012). Speciation of mercury in fish samples by flow injection catalytic cold vapour atomic absorption spectrometry Analytica Chimica (721) 22-27 